\title{
Reflections on Study Abroad: Insights from Registered Nurses
}

\author{
Marie Dietrich Leurer \\ University of Saskatchewan, marie.dietrichleurer@usask.ca \\ Arlene E. Kent-Wilkinson \\ University of Saskatchewan, Arlene.Kent@usask.ca \\ Janet Luimes \\ University of Saskatchewan, Janet.luimes@usask.ca \\ Lee Murray \\ University of Saskatchewan, lee.murray@usask.ca \\ Vicki Squires \\ University of Saskatchewan, vicki.squires@usask.ca \\ Linda M. Ferguson \\ University of Saskatchewan, linda.ferguson@usask.ca \\ Carmen Dell \\ University of Saskatchewan, carmen.dell@usask.ca
}

Follow this and additional works at: https://qane-afi.casn.ca/journal

Part of the Nursing Commons

\section{Recommended Citation}

Dietrich Leurer, Marie; Kent-Wilkinson, Arlene E.; Luimes, Janet; Murray, Lee; Squires, Vicki; Ferguson, Linda M.; and Dell, Carmen (2020) "Reflections on Study Abroad: Insights from Registered Nurses," Quality Advancement in Nursing Education - Avancées en formation infirmière: Vol. 6: Iss. 1, Article 3.

DOI: https://doi.org/10.17483/2368-6669.1225

This Article is brought to you for free and open access by Quality Advancement in Nursing Education - Avancées en formation infirmière. It has been accepted for inclusion in Quality Advancement in Nursing Education - Avancées en formation infirmière by an authorized editor of Quality Advancement in Nursing Education - Avancées en formation infirmière. 


\section{Reflections on Study Abroad: Insights from Registered Nurses}

\section{Cover Page Footnote}

This research was supported by the Western North Western Region Canadian Association of Schools of Nursing Research Award. 
Globalization has led to increased internationalization of higher education, with many universities including internationalization and global engagement as part of their strategic plan (McBride, 2016). The Organisation for Economic Co-operation and Development (2018) reported international students made up $6 \%$ of postsecondary students in member countries in 2016 . In addition to inbound international students, many universities offer study abroad (SA) opportunities to domestic students in an effort to graduate global citizens with cross-cultural skills and international perspectives (Altbach, 2015; McBride, 2016). International efforts to increase SA opportunities confirm the widely accepted view of its many benefits. The British government established a goal to have at least $20 \%$ of its graduating students SA by 2020 (British Council, 2015). In the United States, the number of students participating in SA tripled in the past 20 years, with $10.9 \%$ of all graduating American undergraduate students in 2016-17 having participated in SA as part of their degree program, including an increase from $4.1 \%$ in $2006-07$ to $7.1 \%$ of students in the health professions in 2016-17 (Institute of International Education, 2018).

Canada's SA numbers have remained relatively stable, despite focused efforts to raise SA rates in comparable countries, leading to calls for a national Canadian strategy to increase the number of students who gain SA experience (Study Group on Global Education, 2017). In 2014, approximately $11 \%$ of Canadian university students participated in SA opportunities during their degree, and $97 \%$ of Canadian universities offered some type of international learning experience (Universities Canada, 2014). A Canadian study found almost half of the 74 nursing education programs surveyed offered SA clinical opportunities (Hoe Harwood et al., 2009).

A substantial body of literature outlines the positive outcomes for SA participants, with benefits including increased cross-cultural sensitivity, personal and professional growth, and enhanced communication skills (Afriyie Asenso et al., 2013; Browne et al., 2015; Kelleher, 2013). A systematic literature review found nursing students' SA experiences facilitated cultural competence skills with three main areas of learning: (1) cultural knowledge base including problem-solving and language skills; (2) personal growth including enhanced self-reliance, confidence, tolerance, and an expanded worldview; and (3) exposure to different nursing practices, which promoted reflective comparisons (Kokko, 2011). Nursing students expect these positive outcomes when contemplating applying for SA opportunities (Browne et al., 2015; KentWilkinson et al., 2015).

While the numerous benefits of SA are evident, there are also challenges to be overcome in attaining these positive outcomes. Expectations play a key role in the cross-cultural adjustment process and perceived outcome of SA, with students frequently having unrealistic expectations (McLeod \& Wainwright, 2009; Mendelson, 2004) resulting in stress and disappointment when the realities fall short (Pitts, 2009). Although a majority of students will eventually work through these stresses, Mendelson (2004) found up to $10 \%$ of participants felt disillusioned when their expectations were not met, affecting how students eventually judged the success of their SA experience (McLeod \& Wainwright, 2009). Afriyie Asenso et al. (2013) found nursing students had unrealistic expectations shaped largely by media portrayals of the SA location. Mendelson (2004) suggested that while some students may be able to gain maximum benefits from their SA experience, others will require intervention by educators to adapt their expectations and guide their interpretation of the realities they will face.

There is a need for research that gathers the experiential wisdom of previous nursing SA participants to facilitate attainment of the full range of positive outcomes possible from SA clinical placement experiences. While there is some literature on the perceptions of recent nursing SA 
participants in a single international location, there are few studies that gather insights across diverse placement sites, faculty facilitators, or timeframes. In addition, there appears to be a gap in research examining the viewpoints of SA participants who have since graduated and are able to reflect on their experience from the perspective of a currently practicing registered nurse ( $\mathrm{RN})$.

From 1998 to 2016, a western Canadian university school of nursing offered SA undergraduate clinical placements in Africa, Asia, Europe, and Australia. Approximately 10\% of nursing students applied to these SA opportunities. The intent of this research was to capture the reflections and recommendations of currently practising RNs who participated in the nursing school's SA program. This research, reflecting the context of a singular Canadian nursing program, gathered long-term SA participant insights from diverse placements (high- or low-resource countries) that allowed students to fulfill requirements for different courses during the third or fourth year of their undergraduate program. Although the findings reflect this particular program, the authors believe the commonalities reported from such diverse SA experiences may offer guidance for other nursing programs.

The findings related to the perceived impact of the SA experiences on current nursing practice are reported in another manuscript. This paper is focused on participant reflections on the most beneficial and challenging aspects of their SA experience, and their recommendations to guide the development and delivery of future undergraduate nursing SA placements shared as survey comments or during individual qualitative interviews.

\section{Methods}

Thorne's (2016) interpretive description (ID) qualitative method guided the research process. The ID approach is used in applied qualitative research when the purpose is to improve the practice of a health discipline by providing practical solutions, rather than creating a theory. This approach is consistent with our research objective to gather the insights from previous SA participants with the ultimate goal of informing SA nursing program design and delivery. Sample sizes of 5 to 30 are typical in ID, with the size depending on the nature of the phenomenon of study and on the attainment of sufficient deep sharing of participant perceptions to answer the research question. Purposive sampling is commonly used in ID recruitment to ensure the participants have knowledge of the phenomenon being studied (Thorne, 2016). In total, $168 \mathrm{RN}$ alumnae, who had participated in a school of nursing's SA program in Australia, Finland, Philippines, South Korea, or Tanzania between 2008 and 2015, were invited to participate in this research via their university email addresses. Some invitees may not have seen the invite due to discontinued use of their university email account postgraduation. Participants were first asked to complete an online survey containing both categorical and open-ended comment questions. Survey respondents were then asked if they would be willing to participate in an individual interview. The interview format varied according to participant preference with seven conducted in-person, four via telephone, and two via Skype. Interviews were audio-recorded and transcribed.

Of the 35 survey respondents, 13 agreed to participate in a semi-structured, individual interview. Characteristics of the survey and interview participants are described in Table 1. Consent was obtained before the beginning of both the survey and the interview. Ethical approval was obtained from the university's Behavioural Research Ethics Board [Beh 15-357]. Open-ended survey questions with comment boxes explored participants' perceptions of their SA experiences and recommendations for future SA offerings. These perceptions were further elicited via interviews conducted by a research assistant with one of two research team members who are 
experienced qualitative researchers present for three early interviews. Interviews typically lasted 45 to 60 minutes. As common answers with rich narratives emerged from survey and interview responses, despite the diversity of SA placements, it became evident that the sample size was sufficient to answer the research question and provide guidance for SA program development and delivery.

\section{Table 1}

Participant Characteristics

\begin{tabular}{|c|c|c|}
\hline Characteristics & $\begin{array}{l}\text { Survey } N=35 \\
n(\%)\end{array}$ & $\begin{array}{l}\text { Interview } N=13 \\
n(\%)\end{array}$ \\
\hline \multicolumn{3}{|l|}{ Participant age } \\
\hline 20-24 years & $5(14)$ & $4(31)$ \\
\hline 25-29 years & $18(51)$ & $5(39)$ \\
\hline 30-34 years & $9(26)$ & $3(23)$ \\
\hline 35-39 years & $1(3)$ & $-(-)$ \\
\hline $40+$ years & $2(6)$ & $1(8)$ \\
\hline \multicolumn{3}{|c|}{ Years practised as registered nurse } \\
\hline $1-2$ years & $11(31)$ & $5(39)$ \\
\hline $3-4$ years & $8(23)$ & $4(31)$ \\
\hline $5-6$ years & $11(31)$ & $2(15)$ \\
\hline $7+$ years & $5(14)$ & $2(15)$ \\
\hline \multicolumn{3}{|l|}{ SA host country } \\
\hline Australia & $11(31)$ & $5(39)$ \\
\hline Philippines & $9(26)$ & $2(15)$ \\
\hline Korea & $2(6)$ & $1(8)$ \\
\hline Tanzania & $7(20)$ & $3(23)$ \\
\hline Finland & $4(11)$ & $2(15)$ \\
\hline Mozambique & $2(6)$ & $-(-)$ \\
\hline \multicolumn{3}{|c|}{ Length of SA placement } \\
\hline 3-4 weeks & $10(29)$ & $3(23)$ \\
\hline 5-6 weeks & $17(49)$ & $6(46)$ \\
\hline 7-11 weeks & $3(9)$ & $-(-)$ \\
\hline $12+$ weeks & $5(14)$ & $3(23)$ \\
\hline Undefined & $-(-)$ & $1(8)$ \\
\hline
\end{tabular}

Survey comments and interview narratives were initially analyzed by the first author, with immersion in the data leading to constant comparison among responses as the initial categories were created from similar ideas consistent with ID (Thorne, 2016). This early category formation led to synthesis as categories were expanded, combined, or subdivided according to emerging 
patterns that arose from the data as a result of this iterative analysis. The initial data analysis with supporting reasoning was reviewed by the second author, whose feedback resulted in both authors agreeing on categorical development and discernment of themes across the narratives. In addition, the emerging categories with supporting anonymized quotations were reviewed by all research team members to assist in final categorization and thematic development.

Several evaluation criteria are used in assessing the credibility of ID qualitative research (Thorne, 2016). First, the research should demonstrate epistemological integrity with the research process (Thorne, 2016). Interpretive description (ID) is an approach consistent with the research purpose of collecting qualitative data from RNs who had participated in SA to gather their reflections and recommendations since this approach produces knowledge that can be applied to health contexts. Second, representative credibility ensures that the reported findings are consistent with the sampling used, including triangulation of data sources (Thorne, 2016). In this study, both survey and interview data were gathered from participants across diverse SA placements spanning an eight-year period. Third, analytic logic should make explicit the researcher's analytical reasoning (Thorne, 2016). This was achieved through an audit trail, data analysis by multiple team members, and the provision of verbatim quotes to support the categories identified. Finally, interpretive authority ensures trustworthiness of the researcher's interpretations, free from personal bias (Thorne, 2016). Although some of the research team members had previously been faculty facilitators on specific SA placements, the analysis was conducted by multiple research team members from different SA placements/timeframes to confirm agreement with the analytic interpretations.

\section{Results}

Participants recalled both the positive and the negative features of their SA experience and reflected on what could be done to improve future SA nursing placements. The main categories identified across the surveys and interviews are presented with supporting narratives. The codes behind each interview quotation indicate the participant's placement country (see Table 2) and transcript number. Survey results were aggregated and thus survey quotations cannot be linked to respondent characteristics.

\section{Table 2}

Placement Countries of Interviewees

\begin{tabular}{ll} 
Coding & Country \\
\hline A & Australia \\
F & Finland \\
K & South Korea \\
P & Philippines \\
T & Tanzania \\
\hline
\end{tabular}

\section{Best Aspects of Study Abroad Experience}

Although many positive personal and professional aspects of SA were expressed in the surveys and interviews, three main themes emerged. The most commonly identified best aspects of SA were the opportunity for cross-cultural learning, relationship building, and exposure to diverse health contexts. 
Cross-cultural learning. Participants valued the unique opportunity to be immersed in another culture as part of their nursing education. Cultural learning was often perceived as the most beneficial feature of their SA experience, as articulated by one participant:

The most valuable part of going on this was experiencing the culture and understanding where they're coming from and challenging my own bias as to what my perception was of folks in that culture. (K1)

For some, it was their first time being a visible minority. As one participant described: "You have a better understanding as to what some people go through, especially the minority groups ... I'm so used to being a majority. It was different" (P2). Similarly, a survey comment highlighted that "we all experienced what it was like to be a minority as Caucasian people. It was unforgettable and I will cherish the experience forever."

Participants embraced the opportunity to increase understanding of other cultural perspectives. One survey respondent shared that "my international practicum had a huge influence on my personal growth. I developed a deeper appreciation for other cultures," while another described how "it was a real eye opener to see how other cultures live. Some preconceived notions were disproven." An interviewee noticed interpersonal communication differences but a shared desired to learn about others:

Everybody always asked me why I was so chubby ... like you'd never be asked this at home. It was just one of those things. But people want to learn as much about you as you want to learn as much about them. (P1)

Relationship building. Participants reported that friendships were often key to their SA experience. Long-term relationships were often developed with the people with whom the nursing students had interacted at the SA placement and/or other students on the same SA. One participant developed friendships with both peers and hosts:

Everyone that we met was so warm and welcoming and so generous to us. They'd just put aside their normal lives to accommodate us and I think we met lots of people there that we still keep in contact with. And even just our group of girls that we went with-I think that we developed some really good friendships. (A2)

In some instances, positive student group dynamics were seen as a critical piece in helping participants to adjust to their new environment as the nursing students supported each other. As one participant described: "We were our support system. So, without each other and for as diverse and different as we were, without each other we just wouldn't be able to have functioned as great as we did" (K1). A survey comment described the long-term relationships that were formed: "The bonds made with my fellow travelers still to this day remain strong as we check in with one another from time to time."

Exposure to diverse health contexts. Differences in population health, health care delivery systems, and the nursing role were noted by participants and compared to their own experiences in Canada; an exercise the students considered important to their learning. For example, several assessed population health status, with one participant noting the importance of social support as a social determinant of health in a poverty context:

I learned what community really is and how important it is to people who are suffering from poverty to have a support system that isn't - I don't know how to say it. It was a system that everyone participated in. It wasn't competitive ... The people who stayed at 
home watched all the kids in the whole community and the people who went to work helped support the whole community. (P1)

Another participant recognized similarities and differences in disease prevalence:

The rates of alcoholism are higher there; like pancreatitis and cirrhosis and those kinds of things. But diabetes rates, very similar, and things like flu season, like pneumonia season, all that stuff is very - cancer rates seemed to be about the same too. (F2)

Critical thinking was promoted as part of assessing the root causes for population groups living in circumstances of disadvantage. As an interviewee noted: "historically, there's very eerie similarities with what happened with the Aboriginal people in Australia and what happened in Canada ... I think that our two countries have dealt with them very differently" (A2). Similarly, a survey respondent described:

I really appreciated being exposed to a country with comparable social and disparity issues. It was beneficial to witness the nuanced differences, to compare and contrast some of the programming offered to Aboriginal Australians to Canadian Indigenous peoples.

Providing nursing care in another health care system highlighted systemic differences and allowed participants to consider best practices. As one participant noted: "There's parts of their health care system that I loved, and there's parts that I was like, oh, they're still doing this? We did this 20 years ago" (F1). Others appreciated the chance to practise basic nursing skills in lowresource contexts while recognizing inequities in the host health care system. For example:

Working on the charity wards was so hard and then they switched me to the private wards. And so that was good to see the charities and the private wards, where people can afford everything. It was good to see nursing at its basics where like you don't have equipment. (P1)

Such experiences made some realize they had taken for granted the infrastructure and resources available at home in Canada:

You have to be very conservative with your supplies and you're very cognizant of what you're using there because you're like "once I'm out of this, I'm out," so you have a better idea than you do when you're working here, because you know you can always get what you need here. (T2)

Finally, the narratives detail assessments of nursing procedures and the overall nursing role as critical experiential learning. The participants were surprised to find the nursing scope of practice was expanded in certain countries but reduced in others. A participant placed in a lowresource country was "surprised by LPNs and RNs completing solo prenatal full assessments and clinical diagnosis and prescribing medication" (T3), while another noted the apparent reduced nursing role in another country:

The nurses there don't insert IVs but they do blood draws. So that's kind of the opposite of how it is here for us. It was also a little bit different in general - the nurse physician interactions between nurses and physicians. I felt that the nurses there had a little less autonomy. (A2) 


\section{Difficult Aspects of Study Abroad Experience}

Participants were asked to recall what they had found challenging in reflecting on their SA experience. Several areas were identified including cultural adjustment, financial burden, placement expectations, and social injustice/inequality. Some of the reported difficult aspects of SA were the reverse of beneficial features experienced.

Cultural adjustment. While exposure to a new culture was appreciated as a unique SA benefit, such exposure and distance from home created a challenge for some who reported homesickness and difficulties understanding or fitting into a new cultural context. One participant struggled with language and shared: "I think the most difficult was the language barrier because sometimes patients would, even if it's something that I could very easily care for them, they didn't want me to care for them because I couldn't speak Finnish" (F2).

Others described their personal challenges adjusting to a new culture. One participant recognized her discomfort with new cultural practices and insightfully shared:

It made me feel very lonely and I actually broke down in church and started crying which was slightly embarrassing [laughs] while everyone else is so happy. And it just made me realize how much I miss my own personal cultural practices, my everyday things that I kind of take for granted and didn't really recognize as being part of my background. (K1)

Likewise, a survey respondent provided another personal example:

I struggled with culture shock. This was the biggest, most eye opening experience I had ever felt and honestly, did not feel prepared even though I was told that I would experience this at some point. It's a different feeling than you would expect, and many people experience it differently. There's no time limit as to when this happens for everyone, but it does and will happen to everyone.

Financial burden. Students in this SA program were required to pay the bulk of the associated costs. For some this was a difficult burden, as they chose to prioritize their SA over other financial commitments. A survey respondent described their struggle and recognized the financial burden prevented others from participating:

I was able to fund raise to help pay for it but also cashed in all my RRSP's at a very bad time. I lost a lot of money and have not yet been able to replace them. Still, worth the opportunity but not everyone could do what I did and I don't feel the opportunity should only be given to those who have the money.

Another lamented the cost of the SA experience while simultaneously managing the routine academic costs of nursing education: "And so that was one thing that deterred people because you had to, it says oh it's only six thousand dollars to go to the Philippines, but you also have to pay your tuition" (P1).

Meeting placement expectations. Some participants struggled to adapt their nursing practice to an unfamiliar health care context and/or to make their SA experience fit with the corresponding academic course from their home university. Thus, stress was a result of trying to meet expectations placed on the students from both their home and SA environment. A participant described the difficulties of managing assignments from the home institution while away on the SA placement: "And so I found that very stressful to try and do assignments, because we also didn't always have internet. And we didn't bring textbooks because we were travelling" (A3). 
Differences in biomedical and pharmacological terminology also made attaining clinical competence more difficult. One participant described the challenges:

It was quite difficult learning the different drug names, for I think all of us, because you're used to calling it one thing and then it's called something completely different and so sometimes in the emergency situations you weren't sure what they wanted you to give. It was awkward in the moment and you'd have to quickly look it up. (A5)

And in some situations, participants perceived that the behavioural and academic expectations of host nurse educators differed from those of their Canadian colleagues. The mode of learning presented a challenge for one participant:

The (nursing) classes they had, it was lots of memorization so you know absolutely everything. So you know everything about the body and everything about how the body can go wrong and all the disease processes and whereas here I felt that our school was ... critical thinking is way more here. Which I liked too. There's benefits to both and flaws to both. (P1)

Another found power differentials between students and nursing instructors, along with teaching styles, different from the Canadian nursing education experience:

I struggled with the whole authority thing ... I had questions about suctioning. I hadn't got a lot of experience back home with it and so I had a few questions about it. And so I felt that when I went to this lady about how to do it, she was very strict and to the point. Which was good I needed to know what to do. But she made a point to do it in front of everybody and it was almost embarrassing for me to be scorned at. (P2)

Social injustice/inequality. Another challenge, particularly for students in SA placements in countries without universal health care, was the introduction to inequitable health care provision compared to their experiences in Canada. Several found the poverty context and the denial of health services to those who could not afford them very troubling. One participant observed:

And it was difficult to see this because it was ... I felt terrible. All I could think of is the people who cannot afford these services. Like where are these people getting care? Do these people even get care? (K1)

Another participant was distressed and felt helpless in a situation where lifesaving care was not provided:

This patient came in and her family couldn't afford to rent a ventilator, so she died right next to a room full of ventilators. That was something that I struggled with. And that was the same as with the instructor-she was frustrated by us because we found it difficult accepting the fact that that's an okay thing to happen. (P1)

Other minor categories of difficulties included dealing with logistics such as housing and transportation issues, and instances of negative SA student group dynamics.

\section{Recommendations for Future Study Abroad}

Participants made recommendations to strengthen the SA program for future nursing students. Recommendations included continuing the SA program, providing clear academic expectations, recognizing the value of home faculty presence, and preparing students. 
Continue to offer study abroad. The majority of participants felt the nursing school should continue to offer, and perhaps increase, SA nursing clinical experiences. As one participant advised: "There wasn't a lot of opportunities given how many people were eligible to apply. I just think maybe opening up, expanding on the program" (K1). Study abroad was seen as a superior way to facilitate cultural competence, with a participant suggesting the program should "just keep offering the international experience to the students. Reading materials about cultural diversity in the book is not the same as being there and experiencing in person" (T3). Many felt the experience was a highlight of their nursing undergraduate education, with a survey respondent writing, "It was the best experience about going to university. I would recommend this experience to anyone."

Clear academic expectations. For some participants, the nature of the SA placement sometimes made it difficult to meet their school's course expectations. Close alignment between the course objectives and the experiences available at the SA site was seen as important to maximize learning. One survey respondent expressed frustration when the placement was inconsistent with course intents: "We spent the majority of our time in rural acute care hospitals. It was meant to be a community clinical placement, and I think it would have been more valuable for us to have community nursing placements." For another participant, reduced opportunities to develop independent practice at the host placement made it difficult to meet course objectives:

Sometimes we couldn't always practice our skills because we literally, it wasn't the same as it is here-we weren't allowed to just be on our own doing stuff ... we let our students go off, assess people and-it always felt that there was always someone for like the bigger things. Like dressing changes or sutures. (A3)

Another participant described difficulties in attaining the required clinical hours within the timeframe of the placement because of a different work schedule:

The only thing that was a bit difficult was getting hours, which I don't know how the [School] of Nursing could do anything about that, but they're not always twelves in Finland. They do mostly eights, some twelves and some fourteens. So I'd often have to be putting my hours together like patchwork. (F2)

Faculty presence. Having faculty from the home institution present at the SA placement was reassuring for some participants, particularly if the faculty member had previous experience at that site, while faculty absence was felt as a deficit in placements that lacked this support. Home faculty should be present at the SA experience according to one participant who recommended "maybe figuring out how we can have an instructor there more frequently because just to help sort of mediate some of those challenges" (A4). Another participant described feeling reassured and supported by home faculty on-site:

I liked that [the instructor] came down with us. She was a sense of home and kind of our understanding. She was like our voice 'cause other than that we would have been under the direction of the Filipino instructors. So it was nice to have her there as our backup and our help if we needed it. (P2)

In addition to the presence of home faculty, a survey respondent felt that ideally this instructor should be very knowledgeable about the international setting:

Try to ensure the instructor has a good understanding of the area the students are going to. Our instructor lived there part time and had been for 30 years. I know that is exceptional, but I believe it was a huge part of how much we gained from our time there. 
Finally, while some participants described peer support as key in helping to debrief, others reported the home faculty played an important role through facilitated discussion and reflection, as well as application of appropriate nursing theory. A participant described the significance of such debriefing:

We had a group that really liked to debrief and focus on how we were handling things and so we focused on that. And when our faculty advisor was there we would have meetings with her too. And she was really, really good at that too. (P1)

Student preparation. The importance of extensive student pre-departure preparation was reinforced in the narratives. Participants commonly described how the realities on the ground were quite different than what was anticipated. Survey respondents perceived it would be helpful if "students have a better sense of where they were going and what to expect" and had "more access to information about the experiences students that have already been have had." Having a previous SA student share their experiences before departure was encouraged to promote the creation of realistic expectations and facilitate cultural adjustment. A participant recommended: "Personal testimonies from the groups that have gone before ... 'bring this,' 'do this,' so it was nice to have those helpful hints - things that you can't just find online" (P1). Similarly, another suggested:

For example, if I came and talked to them about ... my challenges that I faced, and then they might not go into it being so, "Oh everything's going to be perfect and our group's going to be amazing and we're all going to be happy." Just having more realistic expectations. (A4)

In addition to basic information on logistics and safety, participants highlighted key content areas that are critical to a successful SA experience, such as knowledge of divergent nursing practices at the placement site. As one participant explained:

In terms of differences between the scope, if you're sending students to an acute care situation, I think you need to have really clear guidelines for them ... So I think that whoever's coordinating has to have a clear understanding of what the differences are and that needs to be put in writing beforehand so it's clear for the students as well as the staff that we're going to be placed with. (A2)

Group dynamics also emerged as an important aspect of the SA experiences, with some groups forming long-term friendships and others experiencing conflicts. Participants recommended students have a chance to get to know one another as part of pre-departure planning and receive guidance on conflict-resolution processes. The importance of getting to know other students in the placement group was described by a participant:

Just encouraging making the relationships prior, because that was a lot of it. If you don't know anyone at all and everyone already has a travel partner that they're rooming with and doing stuff with, it's going to be a lot harder. (A5)

Similarly, a survey respondent wrote: "I feel time should have been focused on group dynamics and group development prior to going internationally." Another survey respondent suggested team building was required to help prevent conflict: "A focus on building strong groups is necessary as group conflict can create very negative memories for everyone involved." Faculty were seen as critical to providing proactive guidance for potential conflict situations that may arise. The importance of faculty facilitation was highlighted by a participant:

We're still young. We're still figuring out who we are. And then we're away from our family and our support systems so, maybe just having the instructor sort of set an example 
for this is how we're dealing with issues and then, just talking about how we're going to deal with issues before they even come up. (A4)

Finally, participants felt it was critical to include cultural adjustment in the pre-departure orientation, although some recognized culture shock cannot be entirely prevented. As one participant shared: "You can't prepare yourself for the experiences that you're going to have or the culture shock that you're going to have. You read about stuff and you think you know it-it just kind of is what it is" (P2). Preparation that helped students adapt and be comfortable in new, diverse situations was seen as a key factor in dealing with cultural adjustment, as one participant described:

So, you need to be able to be prepared to be away from home and out of your comfort element 'cause if not, you're not getting the experience out of it. You're just focused on yourself and getting home and preoccupied by those thoughts too much to be actually able to be enriched by the experience. (T2)

\section{Discussion}

The narratives recall both the beneficial and challenging features of SA across diverse undergraduate nursing placements. Recommendations from the participants provide information on focus areas this program should consider in the design and delivery of future SA experiences.

The positive features of their SA experiences recalled by the RNs are consistent with previous research. First, a desire to learn about other cultures and develop cultural competence is a frequently cited SA goal (Kent-Wilkinson et al., 2015; Kulbok et al., 2012). Kelleher's (2013) integrative review found increased sensitivity to other cultures, beliefs, and values is a typical outcome for SA nursing students. As in our study, this objective is frequently met. Second, relationship building was an important takeaway from their SA for the RNs in our study. This finding was somewhat surprising as friendship formation is not typically mentioned in the literature as an anticipated benefit from SA. Similarly, Philips et al. (2017) found returning SA nursing students viewed the personal international relationships they had developed as an unexpected outcome and expressed the desire to continue to grow these friendships. Finally, the anticipated benefit of gaining international nursing experience (Kent-Wilkinson et al., 2015) was achieved for our participants who strongly valued their exposure to a different health care context. Increased awareness of different nursing roles and health care system practices are also valued outcomes of SA in the literature (Browne et al., 2015; Kelleher, 2013; Kulbok et al., 2012).

Several challenges were identified as participants recalled their SA experience. Many described difficulties with cultural adjustment. Much of the SA research highlights the importance of facilitating student awareness of the cross-cultural adjustments they will experience (Kulbok et al., 2012; McLeod \& Wainwright, 2009; Mendelson, 2004; Pitts, 2009; Zhou et al., 2008). Although SA nursing experiences can increase participant understanding of health system differences and global health issues (Gower et al., 2016), students must be prepared for cultural adaptation and learning in unfamiliar health care systems (Kulbok et al., 2012). Zhou et al. (2008) described the ABC (affective, behavioural, and cognitive) theoretical framework of cultural adaption. Participants in this more contemporary view of cultural adjustment are seen as proactive in their attempts to respond to problems arising from change rather than as the traditional victims of culture shock. Thus, the acculturation process is facilitated through the development of stress coping strategies and culture-specific skills acquisition, with pre- and post-departure preparation of both faculty and students playing an important role. As reinforced by our findings, advice and 
insights from SA alumni is encouraged as an effective way to help shape student expectations and to share adaptation learnings (Hockersmith \& Newfields, 2016).

Additionally, a lack of congruence between the course requirements and SA placement realities was a challenge identified by some participants. Other international exchange programs have reported similar implementation challenges with curricular inflexibility that may not adapt well to differences between program requirements and teaching approaches (Mill et al., 2010).

Participants recommended robust pre-departure preparation. The broader literature outlines the need for comprehensive and reflective pre-departure training as critical in assisting students to develop realistic expectations, create co-student relationships, and develop the knowledge and strategies to deal with stressors common to SA placements. Many university pre-departure programs for SA students are limited to logistics (e.g., travel and safety) and language issues, with a heavy reliance on lecture delivery (Hockersmith \& Newfields, 2016). Opportunities for students to reflect on their goals and to interact with each other are often lacking, suggesting the need to facilitate development of the knowledge and skills necessary to adjust to unique international placements and promote individualized self-reflection on SA expectations (Browne et al., 2015; Burgess et al., 2014; Hockersmith \& Newfields, 2016). For clinical placements, it is particularly important for students to understand the clinical skills that will be required and the host health care, sociocultural, economic, and political contexts (Burgess et al., 2014; Gower et al., 2016).

Exposure to poverty and health care inequities was emotionally upsetting for some participants. Nursing students in other SA programs in low-resource countries have similarly reported the need to negotiate ethical dilemmas (Harrison et al., 2016). With guidance, examination of such ethical issues can lead to transformational learning through critically examining own assumptions and considering other perspectives, including the realities of the host context (Caldwell \& Purtzer, 2014). Increased social consciousness acquired from SA can help to heighten understanding of the social determinants of health through critical self-reflection and moral questioning. Reimer-Kirkham et al. (2009) found nursing students often acquired increased awareness of social injustice as part of SA, and they proposed strategies for nurse educators to maximize this transformative learning and sustain this expanded social consciousness in future nursing practice.

Participants recommended home faculty presence at the international placement. Faculty can play a critical role in helping students to make sense of their experience and promoting critical thinking. Burgess et al. (2014) found SA nursing students may approach their experience from a more paternalistic soft global citizenship perspective rather than a more critical social justice perspective (Andreotti, 2014). Faculty guidance can facilitate development of critical literacy as students move from self-awareness and reflection toward a self-reflexivity that "emphasizes the connections between language, knowledge, power and subjectivities" (Andreotti, 2014, p. 34). Such reflexivity may also assist students to make sense of ethical dilemmas that may arise as part of their SA experience (Caldwell \& Purtzer, 2014).

\section{Implications for Practice}

Given their willingness to be interviewed for this research, as might be expected, these RN participants were strong advocates for undergraduate nursing SA programs. Their descriptions of their lived SA experiences and recommendations have highlighted several key program design and implementation elements that should be considered. 
At the program level, it is important that there be a strong fit between the curriculum and the goals of the SA program. Mill et al. (2010) called for social responsibility and global citizenship to be integrated into undergraduate nursing curricula so all nursing students are exposed to these critical worldviews. Curricular changes at our nursing school include a second-year course that explores the social determinants of health and social justice from a local to global context, and a fourth-year course that applies critical social theory to examine global health and the impact of socio-political policies, health resource allocation, and globalization on human rights. The curricular timing of any SA placement experiences should consider the importance of facilitating the development of critical global citizenship before international experiences.

Another important element is to ensure SA nursing education programs meet relevant ethical standards. Lasker et al. (2018) conducted a scoping review of program guidelines for shortterm global health activities. The aggregated guidelines revealed consensus on six core principles that can provide guidance for ethical and effective SA undergraduate nursing placements. Similarly, McDermott-Levy et al. (2018) consulted with global health nurse experts in developing 10 ethical principles for global health nursing practices that can be applied for SA experiences to low- or middle-income countries. Other program-level considerations include seeking sufficient program resources to provide financial assistance for students who face prohibitive financial barriers to SA participation, ensuring home faculty presence for most/all of the SA experience, and implementing thorough pre-departure preparation.

\section{Future Research}

Several areas of future research would help inform the design and delivery of SA undergraduate nursing programs. The bulk of the literature is focused on the visiting SA nursing student experience, with only occasional gathering of the perspectives of the host placements, particularly in low-resource countries. There is a need for increased research on the perspectives of both parties in SA placements, as well as the promotion of two-way reciprocal SA exchanges. Additionally, research into effective pre-departure SA preparation strategies specific to nursing clinical placements would provide guidance to nurse educators as they strive to help nursing students' better benefit from the exceptional learning opportunities inherent in SA experiences and apply this learning to their own nursing practice.

\section{Limitations}

There were several noteworthy limitations of this study. First, it is possible that only RNs with more positive memories of their SA experience chose to participate. Second, while gathering long-term impressions from SA experiences that had occurred between one to eight years previously was the intent, participants may have forgotten details of their experience. Finally, while participants were involved in SA experiences in diverse placement sites to fulfill requirements of different courses, they were all part of the same school of nursing's SA program, so it cannot be assumed the findings are broadly generalizable to other nursing programs.

\section{Conclusion}

This study captured longer-term reflections of participants in one school of nursing's SA undergraduate program from the view of currently practising RNs. The findings provide an overview of the perceived beneficial and challenging aspects across a variety of diverse international clinical placements over an eight-year timeframe as recalled by the SA participants. Their recommendations for future SA offerings provide guidance for this specific program and 
may have applicability for the planning and delivery of similar undergraduate nursing SA programs. Social responsibility and critical global citizenship should be encouraged as part of the curriculum for all undergraduate nursing students. Additionally, those participating in SA experiences require thorough preparation on the expectations and realities of the SA placement, with faculty guidance to promote advancement toward critical literacy within the context of their international experience. 


\section{References}

Afriyie Asenso, B. A., Reimer-Kirkham, S., \& Astle, B. (2013). In real time: Exploring nursing students' learning during an international experience. International Journal of Nursing Education Scholarship, 10(1), 227-236. https://doi.org/10.1515/ijnes-2012-0045

Altbach, P. G. (2015). Perspectives on internationalizing higher education. International Higher Education, 27, 6-8. https://doi.org/10.6017/ihe.2002.27.6975

Andreotti, V. (2014). Critical and transnational literacies in international development and global citizenship education. Journal of Education, 2(3), 32-50. https://doi.org/10.25749/sis.6544

British Council. (2015). Broadening horizons: The value of the overseas experience. https://www.britishcouncil.org/sites/default/files/6.3 broadening-horizons-2015.pdf

Browne, C., Fetherston, C., \& Medigovich, K. (2015). International clinical placements for Australian undergraduate nursing students: A systematic thematic synthesis of the literature. Nurse Education Today, 35, 1028-1036. https://doi.org/10.1016/j.nedt.2015.05.012

Burgess, C., Reimer-Kirkham, S., \& Astle, B. (2014). Motivation and international clinical placements: Shifting nursing students to a global citizenship perspective. International Journal of Nursing Education Scholarship, 11(1), 75-82. https://doi.org/10.1515/ijnes2013-0056

Caldwell, P., \& Purtzer, M. A. (2014). Long-term learning in a short-term study abroad program: “Are we really truly helping the community?" Public Health Nursing, 32(5), 577-583. https://doi.org/10.1111/phn.12168

Gower, S., Duggan, R., Dantas, J. A., \& Boldy, D. (2016). Motivations and expectations of undergraduate nursing students undertaking international clinical placements. Journal of Nursing Education, 55(9), 487-494. https://doi.org/10.3928/01484834-20160816-02

Harrison, J., Logar, T., Le, P., \& Glass, M. (2016). What are the ethical issues facing globalhealth trainees working overseas? A multi-professional qualitative study. Healthcare, 4, 43. https://doi.org/10.3390/healthcare4030043

Hockersmith, A., \& Newfields, T. (2016). Designing study abroad pre-departure trainings. RYUGAKU: Explorations in Study Abroad, 9(1), 2-12. http://www.tnewfields.info/Articles/PDF/SAResearch.pdf

Hoe Harwood, C., Reimer-Kirkham, S., Sawatzky, R., Terblanche, L., \& Van Hofwegen, L. (2009). Innovation in community clinical placements: A Canadian survey. International Journal of Nursing Education Scholarship, 6(1), Article 28. https://doi.org/10.2202/1548$\underline{923 X .1860}$

Institute of International Education. (2018). Open doors 2018. https://www.iie.org/Research-andInsights/Open-Doors/Open-Doors-2018-Media-Information

Kelleher, S. (2013). Perceived benefits of study abroad programs for nursing students: An integrative review. Journal of Nursing Education, 52(12), 690-695. https://doi.org/10.3928/01484834-20131118-01 
Kent-Wilkinson, A., Dietrich Leurer, M., Luimes, J., Ferguson, L., \& Murray, L. (2015). Study abroad: Exploring factors influencing nursing students' decisions to apply for clinical placements in international settings. Nurse Education Today, 35(8), 941-947. https://doi.org/10.1016/j.nedt.2015.03.012

Kokko, R. (2011). Future nurses' cultural competencies: What are their learning experiences during exchange and studies abroad? A systematic literature review. Journal of Nursing Management, 19(5), 673-682. https://doi.org/10.1111/j.1365-2834.2011.01221.x

Kulbok, P. A., Mitchell, E. M., Glick, D. F., \& Greiner, D. (2012). International experiences in nursing education: A review of the literature. International Journal of Nursing Education Scholarship, 9(1), Article 7. https://doi.org/10.1515/1548-923X.2365

Lasker, J., Aldrink, M., Balasubramaniam, R., Caldron, P. Compton, B., Evert, J., Loh, L., Prasad, S., \& Siegel, S. (2018). Guidelines for responsible short-term global health activities: developing common principles. Globalization and Health, 14, 18. https://doi.org/10.1186/s12992-018-0330-4

McBride, K. (2016). The state of internationalization in Canadian higher education. International Higher Education, 86, 8-9. https://doi.org/10.6017/ihe.2016.86.9362

McDermott-Levy, R., Leffers, J., \& Mayaka, J. (2018). Ethical principles and guidelines of global health nursing practice. Nursing Outlook, 66, 473-481. https://doi.org/10.1016/j.outlook.2018.06.013

McLeod, M., \& Wainwright, P. (2009). Researching the study abroad experience. Journal of Studies in International Education, 13(1), 66-71. https://doi.org/10.1177/1028315308317219

Mendelson, V. G. (2004). "Hindsight is 20/20:" Student perceptions of language learning and the study abroad experience. Frontiers: The Interdisciplinary Journal of Study Abroad, 10, 43-63.

Mill, J., Astle, B. J., Ogilvie, L., \& Gastaldo, D. (2010). Linking global citizenship, undergraduate nursing education, and professional nursing: Curricular innovation in the 21st century. Advances in Nursing Science, 33(3), E1-E11.

Organisation for Economic Co-operation and Development. (2018). Education at a glance 2018: OECD indicators. https://doi.org/10.1787/eag-2018-en

Philips, L., Bloom, T., Gainey, T., \& Chiocca, E. (2017). Influence of short-term study abroad experiences on community health baccalaureate students. Journal of Nursing Education, 56(9), 528-533. https://doi.org/10.3925/01484834-20170817-03

Pitts, M. J. (2009). Identity and the role of expectations, stress, and talk in short-term student sojourner adjustment: An application of the integrative theory of communication and cross-cultural adaptation. International Journal of Intercultural Relations, 33(6), 450 462. https://doi.org/10.1016/j.ijintrel.2009.07.002

Reimer-Kirkham, S., Van Hofwegen, L., \& Pankratz, D. (2009). Keeping the vision: Sustaining social consciousness with nursing students following international learning experiences. International Journal of Nursing Education Scholarship, 6(1), Article 3. https://doi.org/ $\underline{10.2202 / 1548-923 X .1635}$ 
Study Group on Global Education. (2017). Global education for Canadians: Equipping young Canadians to succeed at home \& abroad. Ottawa, ON: Centre for International Policy Studies and Munk School of Global Affairs.

Thorne, S. (2016). Interpretive description: Qualitative research for applied practice (2nd ed.). Routledge.

Universities Canada. (2014). Internationalization at Canadian universities: Quick facts. https://www.univcan.ca/universities/facts-and-stats/internationalization-at-canadianuniversities-quick-facts/

Zhou, Y., Jindal-Snape, D., Topping, K., \& Todman, J. (2008). Theoretical models of culture shock and adaptation in international students in higher education. Studies in Higher Education, 33(1), 63-75. https://doi.org/10.1080/03075070701794833 